\title{
Preparing for the renaissance: treating breast cancer during the COVID-19 pandemic and planning for a safe re-emergence to routine surgical care within a universal health care system
}

\author{
D. Berger-Richardson MD MSc, ${ }^{*}$ G. Ko MD, ${ }^{*}$ and N.J. Look Hong MD MSc${ }^{*+\ddagger}$
}

The evolving COVID-19 pandemic is placing tremendous pressure on health systems. Across Canada and internationally, elective surgeries are being deferred to spare hospital resources, increase system capacity, and limit nosocomial and community spread of the SARS-Cov-2 virus that causes COVID-19. That sudden scale-back in operative resources has inevitably led to delays in cancer surgery. However, longer delays to surgery might be associated with worse disease-specific and overall survival (os) for patients with breast cancer (BCa) ${ }^{1}$. An analysis of the U.S. Surveillance, Epidemiology, and End Results-Medicare database demonstrated that each 30-day increment between diagnosis and surgery for invasie BCa was associated with an os hazard ratio of 1.09 (95\% confidence interval: 1.06 to $1.13 ; p<0.001)^{2}$.

The Ontario Wait Time Strategy recommends that most patients with BCa should undergo surgery within 28 days of a decision to operate. Between October 2019 and December $2019,92 \%$ of women with BCa in Ontario had their surgery within the targeted time period, with a median wait time of 17 days $^{3}$. With the current reduction in operating room (OR) resources because of COVID-19, medical societies, provincial health organizations, and health networks have generated treatment recommendations to help clinicians maximize patient safety during these unprecedented times ${ }^{4-6}$. As a mitigating strategy, most patients will receive neoadjuvant therapy for as long as surgical delays persist (Figure 1).

In the present article, we summarize current treatment recommendations and precautions for BCa management, estimate the burden of accumulating untreated disease, and explore strategies to safely and gradually reintroduce surgical management once oR resources become available. We acknowledge that, across Canada, intra- and interprovincial variation exists with respect to current and evolving surgical supports. However, we believe that common challenges blur geographic borders and motivate our BCa community to maintain the guiding principles of safe and equitable patient care within the confines of our universal health system.

\section{CURRENT TREATMENT RECOMMENDATIONS}

During these times, ongoing multidisciplinary discussions should be generously considered to guide treatment decisions and optimize patient safety. Additionally, frequent communication with patients and families, and offers of psychosocial support, might help to offset inevitable frustrations resulting from surgical delays.

\section{Ductal Carcinoma In Situ}

Compared with patients having invasive cancer, those with ductal carcinoma in situ (DCIS) will likely experience longer delays to surgery. Even when operating at full capacity, there is equipoise with respect to whether active surveillance is safe for low- and intermediate-grade disease, with surgery undertaken only on progression. International trials are currently ongoing ${ }^{7-9}$, with results as yet unpublished. We therefore lack level 1 data to support the oncologic safety of delays in the surgical treatment of DCIS.

Ward et al. ${ }^{10}$ conducted a retrospective review of prospectively collected data from the U.S. National Cancer Database. Increasing delay to surgery for biopsy-proven DCIS was, per 30-day increment, an independent predictor of invasion identified at surgery, but the effect of incremental delay was small (odds ratio: 1.13; $95 \%$ confidence interval: 1.11 to 1.15). Small declines in os were observed for women who experienced delay of more than 6 months between diagnosis and surgery (hazard ratio: 1.12; 95\% confidence interval: 1.09 to 1.16 ), but retrospective analysis prevents a definitive attribution of that result to the delay itself, a combination with intervening factors, or selection bias ${ }^{10}$.

Neoadjuvant endocrine therapy (NET) in estrogen receptor-positive (ER+) DCIS was studied by a group at the University of California-San Francisco. Patients with a biopsy showing DCIS were treated with neoadjuvant letrozole or tamoxifen for 3 months before tumour excision. Posttreatment pathology, compared with the pretreatment core-needle biopsy, showed evidence of DCIs regression and 


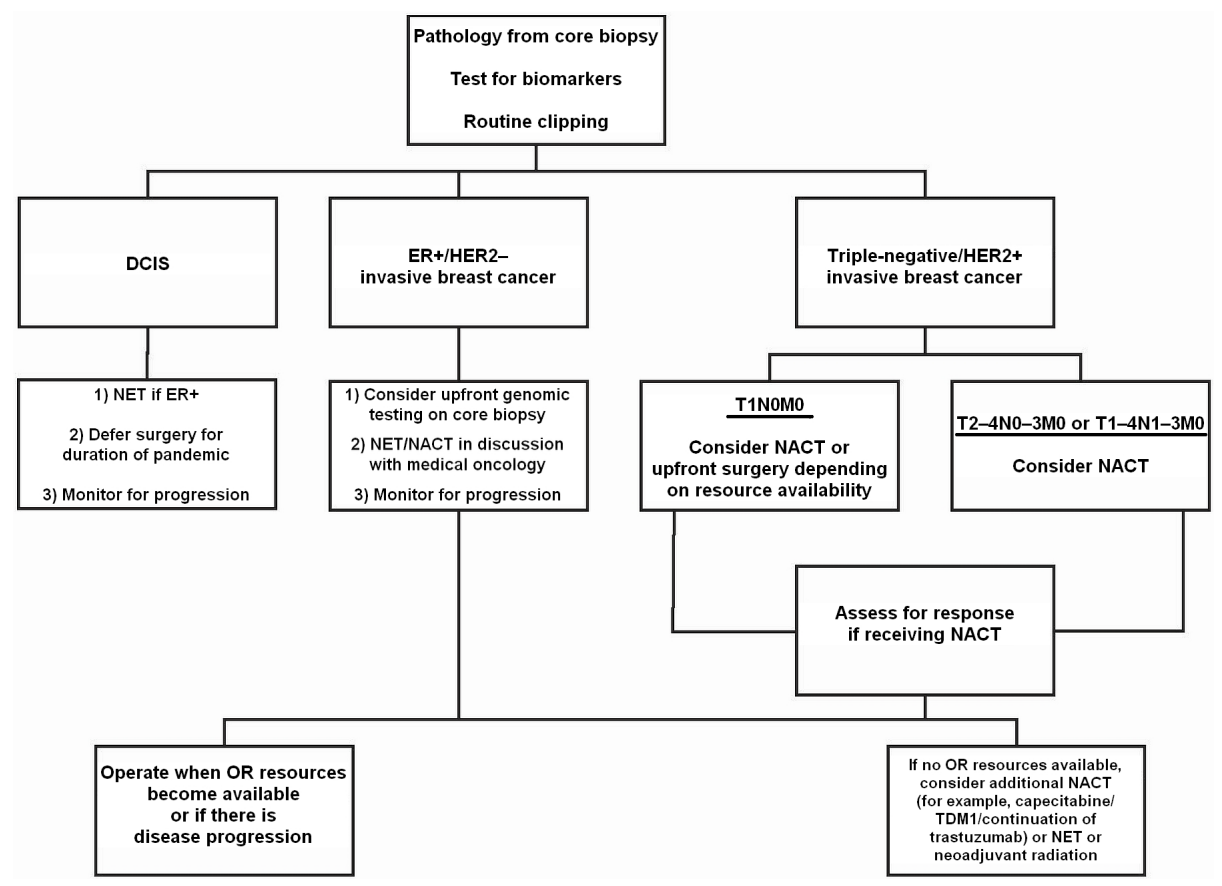

FIGURE 1 Flowchart of breast cancer management during pandemic. DCIS = ductal carcinoma in situ; $E R=$ estrogen receptor; HER2 = human epidermal growth factor receptor 2; NET = neoadjuvant endocrine therapy; NACT = neoadjuvant chemotherapy.

decreased proliferation index $(\mathrm{Ki}-67)^{11}$. The Cancer and Leukemia Group B 40903 Alliance trial similarly studied preoperative letrozole in postmenopausal women with ER+ DCIS. After 6 months of treatment, woman had significantly less enhancement on magnetic resonance imaging and a reduction in Ki-67 score ${ }^{12}$. The foregoing studies demonstrate that $\mathrm{ER}+\mathrm{DCIS}$ might respond to endocrine treatment and, importantly, that the risk of progressing is low.

Overall, the evidence suggests that most patients will not be adversely affected by a delay in surgical treatment of DCIS, particularly with the use of NET. However, the risk of upstaging DCIS to invasive ductal carcinoma after excision persists, ranging from $12 \%$ to $21 \%{ }^{10,12}$. Once OR availability returns, patients with DCIs that is high-grade or palpable should be triaged ahead of those with more favourable histology or those responding to NET. If long delays are anticipated, patients should be monitored every $3-6$ months clinically and radiographically using the imaging modality that best saw the index lesion. Patients experiencing disease progression during the delay should be triaged for urgent intervention.

\section{ER+, HER2-Negative Invasive BCa}

Patients with hormone receptor-positive, HER2 (human epidermal growth factor receptor 2)-negative BCa have primarily been treated with NET in 4 scenarios:

Locally advanced disease

1 A large tumour and a desire for breast-conserving surgery

Presence of frailty or comorbidity and absence of a definite plan for surgery

- Excessive delay before surgery
The current pandemic has led to increased use of NET.

Patients receiving tamoxifen who have additional risk factors for perioperative deep vein thrombosis should discontinue therapy with NET 2-3 weeks before surgery and should receive in-hospital deep vein thrombosis prophylaxis $^{13}$. Tamoxifen is also associated with microvascular flap complications in patients receiving microvascular breast reconstruction (for example, deep inferior epigastric perforator flap $)^{14}$.

In patients with ER+ BCa who receive neoadjuvant chemotherapy (NACT) or NET, the rate of pathologic complete response is less than $10 \%{ }^{15}$. Surgery cannot be delayed indefinitely, and studies have shown that longer delays to surgery are associated with worse disease-specific survival and ${ }^{2}{ }^{2}$. The use of neoadjuvant therapies can mitigate the risk somewhat, but patients have to be followed during the delay, and if they show signs of disease progression, surgery should be a priority. However, most patients can remain on NET for many months with ongoing disease response. Dixon et al. $^{16}$ studied extended use of neoadjuvant letrozole, and most patients experienced ongoing tumour shrinkage with up to 2 years of NET. Only $2 \%$ of patients experienced disease progression.

\section{Triple-Negative or HER2-Positive Invasive BCa}

Patients with triple-negative or HER2-overexpressing (HER2+) invasive BCa staged as $\mathrm{T} 2-4 \mathrm{~N} 0-3 \mathrm{M} 0$ or $\mathrm{T} 1-4 \mathrm{~N} 1-3 \mathrm{M} 0$ are typically candidates for chemotherapy with or without trastuzumab and with or without pertuzumab. Recent trials have shown prognostic value and treatment benefit with additional therapies in patients having residual disease after NACT, leading to an increase in its preoperative use $^{17,18}$. Patients with T1N0M0 tumours larger than $1 \mathrm{~cm}$ are 
increasingly receiving NACT, and in the setting of OR unavailability, NACT should be strongly considered. Small T1N0M0 tumours in this category $(<1 \mathrm{~cm})$ are challenging, because surgical pathology often influences the decision for adjuvant chemotherapy. However, if oR delays persist, the toxicity of NACT has to be balanced with the risk of disease progression.

For patients who have completed NACT, surgery is recommended within 4-8 weeks (allows for recovery from toxicity and myelosuppression) to prevent residual disease progression. Such patients should be a priority for surgery. If surgery is delayed for patients with ER+ cancer, endocrine therapy can be initiated. Patients with HER2+ disease can be treated with anti-HER2 therapy, standard treatment being trastuzumab for a total of 1 year.

Extrapolating from studies which found that patients with triple-negative tumours and residual disease after NACT experience a disease-free survival and os benefit from adjuvant capecitabine and, similarly, that patients with residual HER2+ disease benefit from trastuzumab emtansine (T-DM1), using those treatments as a bridge in the setting of delayed surgery can be considered ${ }^{17,18}$. However, the risk of neuropathy with T-DMl after NACT is high, and in patients with a HER2+ tumour, continuing trastuzumab alone is reasonable. Increased rates of thrombocytopenia are also associated with T-DM1, which is an important perioperative consideration ${ }^{19}$.

Patients with tumours that are borderline unresectable for either breast-conserving therapy or mastectomy should be prioritized for surgery ahead of those with a radiographic complete response.

\section{Atypical Lesions, Discordant Biopsies, and Pleomorphic Lobular Carcinoma In Situ}

Patients with atypical lesions (atypical ductal hyperplasia, atypical lobular hyperplasia, flat epithelial atypia, complex sclerosing lesion, papilloma harbouring atypia), pleomorphic lobular carcinoma in situ, or discordant biopsies at risk of upgrade to malignancy should have their imaging and biopsy results carefully reviewed by the surgeon, pathologist, and radiologist to triage their cases. Repeat biopsies can be considered to avoid the potential need for surgical intervention.

In a systematic review of retrospective studies examining the pathologic features of excised lesions with a preoperative core-needle biopsy showing pleomorphic lobular carcinoma in situ, $15.7 \%$ of specimens harboured concurrent DCIS, and $40.5 \%$, concurrent invasive cancer ${ }^{20}$. If the core-needle biopsy demonstrating pleomorphic lobular carcinoma in situ shows that the lesion is hormone receptor-positive, initiating treatment with endocrine therapy might provide benefit. However, the indication for endocrine therapy in lobular carcinoma in situ is for chemoprevention, and little is known about its effect on the index lesion in the setting of a delay to surgery ${ }^{21}$.

\section{Margin Revisions and Completion Axillary Dissections}

\section{High-Risk Disease}

Current pandemic guidelines recommend that patients with high-risk disease should initiate radiation therapy within 16 weeks of completing chemotherapy or surgery, if resources permit ${ }^{4}$. Included in that guideline are patients with inflammatory $\mathrm{BCa}$, node-positive disease, and triple-negative or HER2+ BCa, and patients who have completed NACT. Such patients should be prioritized for additional surgeries, if necessary, to allow for continuity of care with radiation.

\section{Low-Risk Disease}

Patients with early BCa or DCIS who require a margin revision should be reviewed in a multidisciplinary setting to discuss the pathology and management options. Extent and nature of margin involvement (DCIS vs. invasive disease), risk of residual disease, effect of surgery on need for adjuvant radiation, and hospital resources available should be considered.

The International Guidelines on Radiation Therapy for Breast Cancer During the COVID-19 Pandemic recommend the possible omission of radiation therapy for patients undergoing breast-conserving surgery whose disease is ER+, progesterone receptor-positive, HER2-negative, nodenegative, margin-negative, and grade 1 or 2 ; whose age is greater than 65 years; whose tumour is smaller than $3 \mathrm{~cm}$; and who are being treated with endocrine therapy ${ }^{22}$.

\section{Neoadjuvant Radiation}

When on resources are limited, neoadjuvant radiation can be considered for patients who experience disease progression on neoadjuvant systemic treatment, who cannot tolerate systemic treatment, or who have high-risk disease and have completed NACT. However, the increased risk of potential surgical complications should be discussed with the patient and the multidisciplinary team. Although neoadjuvant radiation is not a standard of care in the absence of clinical progression, the literature demonstrates its safety in early-stage $\mathrm{BCa}^{23}$ and locally advanced $\mathrm{BCa}^{24}$.

\section{Estimating the Burden of Accumulating Untreated Patients with BCa}

At the time of writing, attempting to estimate how long the pandemic will limit or resources is challenging. However, given current disruptions, we can expect ongoing challenges in returning to acceptable wait times for BCa surgery in Canada.

Approximately $40 \%$ of the Canadian population lives in Ontario, and that province's data can be used to model the magnitude of the situation. According to estimates made by Ontario Health (Cancer Care Ontario) before the pandemic, 12,000 cases of invasive $\mathrm{BCa}$ are expected to be diagnosed in 2020: 1000 cases each month on average. In reports from 2013-2014, 65\% of the 2 million women in Ontario 50-74 years of age participate in a BCa screening program. Of every 250 patients screened, 1 has invasive cancer, for an expected 173 screen-detected cancers per month. Some women less than 50 and more than 74 years of age participate in screening, and so approximately 300 screen-detected cancers per month can be estimated. Those cancers will not be detected during the pandemic, because routine screening (including high-risk screening) is on hold per a joint statement released by the Canadian Society of Breast Imaging and the Canadian Association of Radiologists ${ }^{25}$. 
Diagnostic imaging is currently being reserved for patients with a new symptom or palpable abnormality.

It is also likely that women are not receiving routine physical exams or other tests that might incidentally detect a breast mass, and so perhaps only 500 of the anticipated 1000 cases are being diagnosed each month during the pandemic. Of all BCa cases, fewer than 5 in 100 are stage IV at diagnosis, and so almost all patients will need surgery at some point.

It is difficult to estimate how many patients diagnosed with $\mathrm{BC}$ during the pandemic have had surgery. According to the Ontario Ministry of Health, cancer surgeries declined by $40 \%$ between 15 March and 12 April 2020 compared with the same period in 2019. Cancers that tend to progress rapidly, that cause symptoms such as obstruction and bleeding, and that have no viable nonsurgical treatment options, are being prioritized. It can therefore be assumed that $\mathrm{BCa}$, a disease that generally does not cause many symptoms and has effective mitigating treatment options, is subject to proportionally more surgical delay than cancers at other sites. If fewer patients than $30 \%$ of those newly diagnosed during the pandemic are assumed to receive surgery, it could be expected that, when or resources become available, approximately 350 patients diagnosed with BCa will have accumulated per month of oR closure. In addition, 1000 new cases each month (plus perhaps more, if imaging or diagnostic capacity is temporarily increased) will be diagnosed into the future. Furthermore, patients diagnosed with cancer in the weeks preceding the pandemic-and a number of patients with DCIS or atypical lesions, and some awaiting additional breast procedures-would not be captured by the foregoing numbers. Table I models the estimated burden of BCa cases requiring surgery in Ontario depending on the pandemic duration and the number of surgeries performed during the reduction in oR resources.

\section{Strategies to Ensure a Safe Surgical Renaissance}

Although the numbers in Table I represent a very crude estimate of the number of patients requiring surgery,

TABLE I Modelled estimates of the cumulative number of breast cancer cases delayed in Ontario because of restrictions on surgical capacitya

\begin{tabular}{|c|c|c|c|c|}
\hline \multirow{2}{*}{\multicolumn{2}{|c|}{ Estimate }} & \multicolumn{3}{|c|}{ Reduction in operating room capacity } \\
\hline & & $80 \%$ & $60 \%$ & $40 \%$ \\
\hline \multicolumn{5}{|c|}{ Cases deferred $(n)$} \\
\hline & After 1 month $^{b}$ & 699 & 524 & 350 \\
\hline & After 2 months ${ }^{\mathrm{c}}$ & 1140 & 855 & 570 \\
\hline & After 3 months $^{\mathrm{c}}$ & 1520 & 1140 & 760 \\
\hline \multicolumn{5}{|c|}{$\begin{array}{l}\text { Assumptions: restrictions persist for } 3 \text { months, rate of restriction is } \\
\text { constant, } 1000 \text { new breast cancer cases are typically diagnosed } \\
\text { per month. Those numbers do not include patients who are not } \\
\text { diagnosed because of lack of screening. }\end{array}$} \\
\hline \multicolumn{5}{|c|}{$\begin{array}{l}\text { surgeries were postponed. Assumes } 950 \text { breast surgeries per month } \\
\text { (1000 breast cancers diagnosed per month - 5\% stage IV cases for } \\
\text { which surgery is not performed), with } 92 \% \text { of eligible surgeries being } \\
\text { scheduled and completed within } 1 \text { month of the decision to operate. }\end{array}$} \\
\hline \multicolumn{5}{|c|}{$\begin{array}{l}\text { Assumes that } 475 \text { stages I-III breast cancers continue to be } \\
\text { diagnosed while imaging is on hold and an equal number of pa- } \\
\text { tients are starting neoadjuvant therapy as others are completing } \\
\text { neoadjuvant therapy. }\end{array}$} \\
\hline
\end{tabular}

they highlight the urgent need for a sustainable surgical recovery strategy. Although nuanced individual provincial approaches will certainly be created for a return to routine care, these principles should be considered:

Elective surgery for benign conditions (for example, fibroadenoma), prophylaxis, and autologous reconstruction should continue to be held while the collection of cancer cases receives priority. As resources grow, a staged approach to reinstate elective services can be considered.

- Cancer patients could continue to be treated with neoadjuvant therapies for an indefinite period as surgeons work with their hospitals to safely increase surgical capacity.

1. Regions could consider organizing designated cancer treatment centres to start operating on a high volume of oncology cases. The resources in those individual centres would exclusively focus on cancer care and not conflict with the intensive resources required for patients having COVID-19. However, streamlined repeat breast imaging and physician consultation might be required. Such a strategy might expose patients to more hospital visits and risk of nosocomial spread.

- A coordinated and dynamic provincial resource ("dashboard") that monitors on availability and untreated cancer patients within each institution would help to facilitate triage and transfer of patients to centres with the greatest capacity. However, such a strategy requires ongoing oversight to ensure the accuracy of the evolving numbers, and streamlined processes at each institution to accept and transfer patients as needed.

\section{FINAL THOUGHTS}

The global Covid-19 pandemic has had a tremendous impact on all facets of society. It has caused enormous shifts in treatment paradigms for patients with BCa. As the reopening of oR resources is contemplated, increased communication and strategic planning between members of the oncology community will be required to safely manage affected patients and to return to pre-pandemic standards of care. The current situation also presents an opportunity to evaluate the oncologic outcomes of patients treated with neoadjuvant therapy who would otherwise have had upfront surgery and to learn from the experience for future consideration.

\section{DISCLAIMER}

The opinions expressed in this publication are those of the authors. They do not purport to reflect the opinions or views of provincial or hospital strategic planning committees.

\section{CONFLICT OF INTEREST DISCLOSURES}

We have read and understood Current Oncology's policy on disclosing conflicts of interest, and we declare that we have none.

\section{AUTHOR AFFILIATIONS}

*Department of Surgery, University of Toronto, ${ }^{\dagger}$ Division of Surgical Oncology, Sunnybrook Health Sciences Centre, and łInstitute of Health Policy, Management and Evaluation, University of Toronto, Toronto, ON 


\section{REFERENCES}

1. Bleicher RJ. Timing and delays in breast cancer evaluation and treatment. Ann Surg Oncol 2018;25:2829-38.

2. Bleicher RJ, Ruth K, Sigurdson ER, et al. Time to surgery and breast cancer survival in the United States. JAMA Oncol M2016;2:330-9.

3. Health Quality Ontario (HQO). Time from decision to having cancer surgery [Web resource]. Toronto, ON: HQO; 2020. [Available at: https://www.hqontario.ca/System-Performance/ Wait-Times-for-Surgeries-and-Procedures/Wait-Times-forCancer-Surgeries/Time-from-Decision-to-Having-CancerSurgery; cited 2 April 2020]

4. American Society of Breast Surgeons (ASBRS). Recommendations for Prioritization, Treatment and Triage of Breast Cancer Patients During the COVID-19 Pandemic: Executive Summary. Ver. 1.0 2020. Columbia, MD: ASBRS; 2020.

5. Society of Surgical Oncology (sso). Resource for Management Options of Breast Cancer during COVID-19. Rosemont, IL: SSO; 2020.

6. Ontario Health (Cancer Care Ontario) [OH(CCO)]. Pandemic Planning Clinical Guideline for Patients with Cancer. Toronto, ON: OH(CCO); 2020. [Downloadable at: https://www. cancercareontario.ca/en/guidelines-advice/types-of-cancer/ 64736; cited 17 June 2020]

7. Elshof LE, Tryfonidis K, Slaets L, et al. Feasibility of a prospective, randomised, open-label, international multicentre, phase III, non-inferiority trial to assess the safety of active surveillance for low risk ductal carcinoma in situ-the LORD study. Eur J Cancer 2015;51:1497-510.

8. Francis A, Thomas J, Fallowfield L, et al. Addressing overtreatment of screen detected DCIS; the LORIS trial. Eur JCancer 2015;51:2296-303.

9. Hwang ES, Hyslop T, Lynch T, et al. The COMET (Comparison of Operative versus Monitoring and Endocrine Therapy) trial: a phase III randomised controlled clinical trial for low-risk ductal carcinoma in situ (DCIS). BMJ Open 2019;9:e026797.

10. Ward WH, DeMora L, Handorf E, et al. Preoperative delays in the treatment of DCIS and the associated incidence of invasive breast cancer. Ann Surg Oncol 2020;27:386-96.

11. Chen YY, DeVries S, Anderson J, et al. Pathologic and biologic response to preoperative endocrine therapy in patients with ER-positive ductal carcinoma in situ. BMC Cancer 2009;9:285.

12. Hwang ES, Hyslop T, Hendrix LH, et al. Phase II single-arm study of preoperative letrozole for estrogen receptor-positive postmenopausal ductal carcinoma in situ: CALGB 40903 (Alliance). J Clin Oncol 2020:38:1284-92.
13. Hussain T, Kneeshaw PJ. Stopping tamoxifen peri-operatively for VTE risk reduction: a proposed management algorithm. Int J Surg 2012;10:313-16.

14. Kelley BP, Valero V, Yi M, Kronowitz SJ. Tamoxifen increases the risk of microvascular flap complications in patients undergoing microvascular breast reconstruction. Plast Reconstr Surg 2012;129:305-14.

15. Spring LM, GuptaA, Reynolds KL, etal. Neoadjuvant endocrine therapy for estrogen receptor-positive breast cancer: a systematic review and meta-analysis. JAMA Oncol 2016;2:1477-86.

16. Dixon JM, Renshaw L, Macaskill EJ, et al. Increase in response rate by prolonged treatment with neoadjuvant letrozole. Breast Cancer Res Treat 2009;113:145-51.

17. von Minckwitz G, Huang CS, Mano MS, et al. Trastuzumab emtansine for residual invasive HER2-positive breast cancer. N Engl J Med 2019;380:617-28.

18. Masuda N, Lee SJ, Ohtani S, et al. Adjuvant capecitabine for breast cancer after preoperative chemotherapy. NEnglJMed 2017;376:2147-59

19. Kowalczyk L, Bartsch R, Singer CF, Farr A. Adverse events of trastuzumab emtansine (T-DM1) in the treatment of HER2-positive breast cancer patients. Breast Care (Basil) 2017; 12:401-8.

20. Wazir U, Wazir A, Wells C, Mokbel K. Pleomorphic lobular carcinoma in situ: current evidence and a systemic review. Oncol Lett 2016;12:4863-8.

21. Trivedi MS, CoeAM, Vanegas A, Kukafka R, Crew KD. Chemoprevention uptake among women with atypical hyperplasia and lobular and ductal carcinoma in situ. Cancer Prev Res (Phila) 2017;10:434-41.

22. Coles CE, Aristei C, Bliss J, et al. International guidelines on radiation therapy for breast cancer during the COVID-19 pandemic. Clin Oncol (R Coll Radiol) 2020;32:279-81.

23. Glynne-Jones R, Tan D, Hughes R, Hoskin P. Squamous-cell carcinoma of the anus: progress in radiotherapy treatment. Nat Rev Clin Oncol 2016;13:447-59.

24. Hughes K, Neoh D. Neoadjuvant radiotherapy: changing the treatment sequence to allow immediate free autologous breast reconstruction. J Reconstr Microsurg 2018;34:624-31.

25. Canadian Association of Radiologists (CAR). Canadian Society of Breast Imaging and Canadian Association of Radiologists Joint Position Statement on COVID-19. Ottawa, ON: CAR; 2020. [Available online at: https://car.ca/news/canadian-societyof-breast-imaging-and-canadian-association-of-radiologistsjoint-position-statement-on-covid-19/\#more-11195; cited 2 April 2020] 